УдК 316.346.32-053.9:314.15

\title{
СРАВНИТЕЛЬНЫЙ АНАЛИЗ СОЦИАЛЬНО-ДЕМОГРАФИЧЕСКИХ ХАРАКТЕРИСТИК ПОЖИЛЫХ ЛЮДЕЙ В РОССИИ
}

\author{
Фролова Елена Александровна, \\ frolova_ea@mail.tsu.ru \\ Кашапова Эльмира Рамисовна, \\ elmira6280@yandex.ru \\ Клемашева Елена Игоревна, \\ klemaha@mail.ru \\ Маланина Вероника Анатольевна, \\ milanskaya@mail.ru
}

Национальный исследовательский Томский политехнический университет, Россия, 634050, пр. Ленина, 30.

\begin{abstract}
Фролова Елена Александровна, доктор экономических наук, профессор кафедры экономики Института экономики и менеджмента Национального исследовательского Томского государственного университета Россия, 634050, пр. Ленина, 30; ведущий научный сотрудник Международной научно-образовательной лаборатории технологий улучшения благополучия пожилых людей Национального исследовательского Томского политехнического университета,.
\end{abstract}

Кашапова Эльмира Рамисовна, лаборант Международной научно-образовательной лаборатории технологий улучшения благополучия пожилых людей Национального исследовательского Томского политехнического университета.

Клемашева Елена Игоревна, кандидат экономических наук, лаборант Международной научнообразовательной лаборатории технологий улучшения благополучия пожилых людей Национального исследовательского Томского политехнического университета.

Маланина Вероника Анатольевна, кандидат экономических наук, доцент отделения социальногуманитарных наук Школы базовой инженерной подготовки Национального исследовательского Томского политехнического университета.

На рубеже XX-XXI вв. в экономической науке и социальной политике проблематика неравенства и бедности вновь начала актуализироваться. Однако чаще всего объектом исследования и адресатами мер по их преодолению становятся граждане страны в целом. Предполагается, что повышение пенсий, социальных выплат, введение прогрессивной налоговой системы приведут к сглаживанию неравенства в доходах. Вместе с тем провоцировать бедность и неравенство могут не только институциональные условия, но и совокупность событий и решений, принимаемых индивидами. Цель данной работы - сравнительный анализ социально-демографических характеристик пожилых людей в России в контексте неравенства по доходам и определение факторов, коррелирующих с их принадлежностью к определенной доходной страте общества. Методы исследования: методы расчета децильных и квинтильных коэффициентов неравенства по доходам, метод расчета индекса Джини и индекса Пальмы для граждан России четырех возрастных категорий от 55 лет и старше. Результаты. Проведенные оценки значений коэффициентов и индексов неравенства позволяют предположить, что с возрастом коэффициенты неравенства внутри совокупности пожилых людей снижаются. Группы наименее и наиболее обеспеченных пожилых людей схожи только тем, что состоят преимущественно из женщин. По остальным параметрам группы имеют существенные отличия (особенно группа людей в возрасте 55-75 лет). Наименее обеспеченные люди 
проживают преимущественно в сельской местности, имеют более низкий уровень образования, занятости, мобильности и низкую оценку собственного здоровья по сравнению с наиболее обеспеченными.

Ключевые слова: Неравенство, доходное неравенство, пожилые люди, индекс Джини, бедность.

\section{Введение}

Изучение неравенства в экономической науке традиционно происходит на базе выявления степени выраженности доходного неравенства [1-4], которое, в свою очередь, измеряется с помощью индекса Джини. Функционал индекса Джини позволяет видеть распределение дохода по разным группам населения и количественно оценивать степень доходного неравенства. Индекс Пальмы отражает соотношение доходов $10 \%$ самой обеспеченной прослойки и 40 \% наименее обеспеченной. Несмотря на эволюцию налоговых систем, систем социальной защиты населения, роста вмешательства государства в процесс перераспределения доходов, нигде в мире распределение доходов не является строго равномерным [5-7]. Наличие некоторого уровня неравенства, напротив, считается фактором, стимулирующим экономический рост в целом, а также инвестиции в человеческий капитал и развитие рынка труда в частности $[8,9]$. Общемировая тенденция старения населения сопровождается увеличением доли пожилых людей в составе населения и вносит поправки в дискуссию о роли неравенства в обществе [10].

В отношении такой специфической группы населения, как пожилые люди, работают не все аргументы в пользу экономического неравенства. И если в раннем пожилом возрасте 55-65 лет для старшего поколения участие в трудовой деятельности продолжает оставаться актуальным, то стимулировать выход на рынок труда в более позднем возрасте - стратегия весьма ограниченного действия. В научной литературе существует гипотеза о «выравнивании» возможностей людей с возрастом и соответствующем снижении неравенства. Данная гипотеза находит подтверждение в ряде исследований $[11,12]$. Сравнение децильных коэффициентов для российских граждан моложе и старше 55 лет также дает основание утверждать, что люди в трудоспособном возрасте более подвержены неравенству по сравнению с пожилыми соотечественниками. Причины бедности и неравенства часто формируются уже на ранних этапах жизни человека и связаны с недостаточным уровнем образования и плохим здоровьем, что не всегда компенсируется в течение жизненного курса (life course) индивида [13-16]. В целом существует понимание того, что в вопросе смягчения доходного неравенства важны как указанные выше стартовые условия, так и институциональная среда - пенсионные и налоговые системы, дизайн которых может предусматривать различную интенсивность перераспределения доходов в рамках национальной экономики от более обеспеченных к менее обеспеченным гражданам.

\section{Методология исследования}

В качестве опорной методологии использован метод расчета децильных и квинтильных коэффициентов. Децильные (квинтильные) коэффициенты являются универсальными показателями дифференциации доходов между 10 \% (и 20 \% соответственно) наименее обеспеченных и 10 \% (и 20 \%) наиболее обеспеченных граждан. Разрыв в доходах двух полярных групп населения и динамика этого разрыва позволяет оценивать степень доходного неравенства и успешность мер государственной политики (при наличии таковой) по его преодолению или сглаживанию (табл. 1). Источниками данных 
для расчетов степени неравенства выступили две последние волны общероссийского Комплексного мониторинга условий жизни населения (КОУЖ) 2016 и 2018 гг., а также официальная статистика по экономическому положению старшего поколения в России $[17,18]$.

Таблица 1. Параметры неравенства среди пожилых людей в России (по данным КОУЖ за 2016 и 2018 г2.)

Table 1. Inequality parameters among older adults in Russia (according to Comprehensive monitoring of living conditions, 2016 and 2018)

\begin{tabular}{|c|c|c|c|c|c|c|}
\hline $\begin{array}{l}\text { Возрастные } \\
\text { группы } \\
\text { Age groups }\end{array}$ & $\begin{array}{c}\text { Временные } \\
\text { рамки } \\
\text { Time period }\end{array}$ & $\begin{array}{c}\text { Количество рес- } \\
\text { пондентов в вы- } \\
\text { борке } \\
\text { Number of re- } \\
\text { spondents in the } \\
\text { sample } \\
\end{array}$ & $\begin{array}{c}10 \% / 10 \% \text { (де- } \\
\text { цильный коэффи- } \\
\text { циент) } \\
10 \% / 10 \% \\
\text { (decile ratio) } \\
\end{array}$ & $\begin{array}{c}20 \% / 20 \% \\
\text { (квинтильный ко- } \\
\text { эффициент) } \mid 20 \\
\% / 20 \% \\
\text { (quintile coeffi- } \\
\text { cient) } \\
\end{array}$ & \begin{tabular}{|c|}
$10 \% / 40 \%$ \\
(Индекс \\
Пальмы) \\
$10 \% / 40 \%$ \\
(Palm \\
Index) \\
\end{tabular} & $\begin{array}{c}\text { Индекс } \\
\text { Джини } \\
\text { Gini } \\
\text { Index }\end{array}$ \\
\hline \multirow{3}{*}{$\begin{array}{c}\text { Итого (55+) } \\
\text { Total (55+) }\end{array}$} & 2016 & 36166 & 4,65 & 3,195 & 3,170 & 22,2 \\
\hline & 2018 & 37221 & 4,22 & 2,954 & 3,140 & 20,7 \\
\hline & $2018 / 2016$ & $2,92 \%$ & $-0,427$ & $-0,240$ & $-0,030$ & $-1,47$ \\
\hline \multirow{3}{*}{$55-65$} & 2016 & 19538 & 6,682 & 4,452 & 4,422 & 29,2 \\
\hline & 2018 & 18933 & 6,235 & 4,087 & 4,204 & 28,2 \\
\hline & $2018 / 2016$ & $-3,10 \%$ & $-0,447$ & $-0,364$ & $-0,218$ & $-0,97$ \\
\hline \multirow{3}{*}{$66-75$} & 2016 & 9998 & 4,438 & 3,061 & 3,085 & 21,8 \\
\hline & 2018 & 11284 & 4,191 & 2,882 & 3,027 & 21,15 \\
\hline & $2018 / 2016$ & $12,86 \%$ & $-0,247$ & $-0,179$ & $-0,058$ & $-0,68$ \\
\hline \multirow{3}{*}{$76-85$} & 2016 & 5697 & 3,779 & 2,610 & 2,531 & 18,48 \\
\hline & 2018 & 6006 & 3,264 & 2,449 & 2,444 & 17,27 \\
\hline & $2018 / 2016$ & $5,42 \%$ & $-0,515$ & $-0,160$ & $-0,086$ & $-1,20$ \\
\hline \multirow{3}{*}{$85+$} & 2016 & 933 & 3,705 & 2,655 & 2,642 & 19,2 \\
\hline & 2018 & 998 & 3,205 & 2,398 & 2,884 & 16,26 \\
\hline & $2018 / 2016$ & $6,97 \%$ & $-0,499$ & $-0,257$ & 0,241 & $-3,03$ \\
\hline
\end{tabular}

Данные свидетельствуют о некотором снижении значений децильных, квинтильных коэффициентов и индекса Джини для всех возрастных групп пожилых людей в России в 2018 г. по сравнению с 2016 г. Индекс Пальмы демонстрирует аналогичную динамику, за исключением самой пожилой подгруппы 85+. Следует отметить, что индекс Джини для России (для граждан всех возрастов) составил 41,4 в 2016 г. и 41,1 в 2018 г. $[19,20]$.

Для формулировки мер по преодолению или смягчению степени неравенства и бедности необходимо представлять социально-демографический портрет типичного адресата подобных мер. По данным КОУЖ этот портрет выглядит следующим образом для четырех возрастных подгрупп (табл. 2). 
Таблища 2. Социально-демографические характеристики наименее обеспеченных (10\%-1) и наиболее обеспеченных (10\%-10) пожилых людей в России (по данным КОУЖ за 2016 г.)

Table 2. Social and demographic characteristics of the least (10\%-1) and the most (10\%-10) wealthy older adults in Russia (according to Comprehensive monitoring of living conditions, 2016)

\begin{tabular}{|c|c|c|c|c|c|c|c|c|}
\hline \multirow{2}{*}{\begin{tabular}{|l|}
\multicolumn{1}{|c|}{ Возрастные группы } \\
\multicolumn{1}{|c|}{ Age groups } \\
Характеристики \\
Characteristics \\
\end{tabular}} & \multicolumn{2}{|c|}{$55-65$} & \multicolumn{2}{|c|}{$66-75$} & \multicolumn{2}{|c|}{$76-85$} & \multicolumn{2}{|c|}{$85+$} \\
\hline & $10 \%-1$ & $10 \%-10$ & $10 \%-1$ & $10 \%-10$ & $10 \%-1$ & $10 \%-10$ & $10 \%-1$ & $10 \%-10$ \\
\hline $\begin{array}{l}\text { доля женщин, \% } \\
\text { share of women, \% }\end{array}$ & 61,26 & 54,64 & 72,24 & 58,10 & 77,27 & 66,02 & 74,19 & 58,33 \\
\hline $\begin{array}{l}\text { доля городских жителей, \% } \\
\text { share of urban residents, \% }\end{array}$ & 38,02 & 84,62 & 44,19 & 85,20 & 38,46 & 81,95 & 56,99 & 70,83 \\
\hline $\begin{array}{l}\text { средний возраст, лет } \\
\text { average age, years }\end{array}$ & 59,5 & 59,3 & 69,56 & 69,3 & 78,7 & 80,15 & 87,9 & 89,35 \\
\hline $\begin{array}{l}\text { не состоят в браке, } \% \\
\text { not married, \% }\end{array}$ & 27,58 & 14,97 & 52,91 & 38,00 & 71,68 & 69,20 & 80,65 & 83,33 \\
\hline $\begin{array}{l}\text { проживают совместно с супру- } \\
\text { гом(ой), \% } \\
\text { live together with spouse, } \%\end{array}$ & 53,63 & 63,35 & 36,37 & 50,60 & 24,13 & 26,19 & 19,35 & 14,58 \\
\hline $\begin{array}{l}\text { образование не выше основного } \\
\text { общего, \% } \\
\text { education is not higher than the basic } \\
\text { general, \% }\end{array}$ & 13,66 & 1,38 & 33,22 & 6,4 & 63,11 & 33,98 & 77,42 & 46,88 \\
\hline $\begin{array}{l}\text { средний доход на члена домохо- } \\
\text { зяйства (р. в мес.) } \\
\text { average income per household mem- } \\
\text { ber (rubles per month) }\end{array}$ & 6112 & 40846 & 6958 & 30884 & 6958 & 26302 & 8228 & 30486 \\
\hline $\begin{array}{l}\text { не малоимущие, \% } \\
\text { not poor, \% }\end{array}$ & 12,38 & 100 & 32,57 & 100 & 68,01 & 100 & 53,76 & 100 \\
\hline $\begin{array}{l}\text { дети, проживающие отдельно, } \\
\text { оказывают денежную помощь, \% } \\
\text { children living separately provide } \\
\text { cash assistance, \% }\end{array}$ & 30,50 & 12,15 & 36,37 & 35,90 & 36,71 & 25,84 & 34,41 & 19,79 \\
\hline $\begin{array}{l}\text { дети, проживающие отдельно, } \\
\text { покупают продукты, одежду, \% } \\
\text { children living separately buy food, } \\
\text { clothes, \% }\end{array}$ & 28,10 & 15,48 & \begin{tabular}{|l|}
39,88 \\
\end{tabular} & 28,50 & 45,45 & 50,97 & 59,14 & 53,13 \\
\hline $\begin{array}{l}\text { получатели пенсии по возрасту, \% } \\
\text { recipients of old-age pension, \% }\end{array}$ & 71,44 & 59,97 & 92,48 & 74,90 & 95,98 & 85,13 & 90,32 & 82,29 \\
\hline $\begin{array}{l}\text { не относятся к получателям феде- } \\
\text { ральных льгот, \% } \\
\text { not recipients of federal benefits, \% }\end{array}$ & 89,05 & 95,18 & 85,47 & 88,00 & 82,69 & 62,83 & 67,74 & 33,33 \\
\hline $\begin{array}{l}\text { не относятся к получателям ре- } \\
\text { гиональных льгот, \% } \\
\text { not recipients of regional benefits, \% }\end{array}$ & 61,00 & 42,90 & 43,99 & 26,20 & 35,84 & 25,66 & 23,66 & 23,96 \\
\hline $\begin{array}{l}\text { никогда не совершали туристиче- } \\
\text { скую поездку, \% } \\
\text { never made a tourist trip, \% }\end{array}$ & 67,25 & 17,84 & 63,33 & 23,50 & 70,98 & 34,69 & 75,27 & 43,75 \\
\hline $\begin{array}{l}\text { не ездили никуда по причине от- } \\
\text { сутствия денег, \% } \\
\text { did not go anywhere due to lack of } \\
\text { money, \% }\end{array}$ & 49,59 & 7,59 & 42,89 & 11,00 & 29,37 & 7,96 & 15,05 & 4,17 \\
\hline
\end{tabular}


Окончание табл. 2

Table 2

\begin{tabular}{|c|c|c|c|c|c|c|c|c|}
\hline $\begin{array}{l}\text { неработающие, \% } \\
\text { not employed, \% }\end{array}$ & 80,30 & 21,63 & 96,89 & 63,50 & 99,65 & 95,58 & 100 & 98,96 \\
\hline $\begin{array}{l}\text { самооценка состояния здоровья } \\
\text { как плохого, \% } \\
\text { poor self-perceived health, \% }\end{array}$ & 16,53 & 6,97 & 28,96 & 19,70 & 46,85 & 43,72 & 49,46 & 42,71 \\
\hline $\begin{array}{l}\text { не ездили никуда по состоянию } \\
\text { здоровья, \% } \\
\text { did not go anywhere for health rea- } \\
\text { sons, \% }\end{array}$ & 6,14 & 2,41 & 12,53 & 10,10 & 30,42 & 31,68 & 46,24 & 43,75 \\
\hline $\begin{array}{l}\text { диспансерное наблюдение, \% } \\
\text { dispensary observation, \% }\end{array}$ & 44,83 & 36,96 & 57,52 & 55,30 & 60,14 & 73,98 & 54,84 & 79,17 \\
\hline $\begin{array}{l}\text { отсутствие инвалидности, \% } \\
\text { no disability, \% }\end{array}$ & 87,10 & 94,36 & 83,37 & 87,30 & 79,20 & 59,47 & 69,89 & 43,75 \\
\hline $\begin{array}{l}\text { итого респондентов, чел. } \\
\text { total respondents, people }\end{array}$ & 1954 & 1951 & 998 & 1000 & 572 & 565 & 93 & 96 \\
\hline
\end{tabular}

Анализ данных Комплексного обследования условий жизни населения за 2016 год показал, что среди 10 \% наименее обеспеченных граждан (группа 10 \%-1) преобладают жители сельской местности (за исключением возраста 85+, в котором среди малообеспеченных граждан с небольшим перевесом преобладают горожане). Среди граждан с низкими доходами во всех пожилых возрастах преобладают женщины, их в среднем более $70 \%$. Помощь со стороны детей данной категории пожилых людей весьма ограничена: в среднем 35 \% пожилых респондентов дети помогают деньгами, 43 \% покупают продукты и одежду, причем денежная помощь не зависит от возраста, а натуральная помощь через покупку продуктов и вещей имеет тенденцию к росту с увеличением возраста реципиентов. Отсутствие работы является существенным и практически универсальным признаком 10 \% наименее обеспеченных пожилых людей в России. Даже в самой молодой группе пожилых 55-65 лет менее $20 \%$ респондентов имеют работу, а в более старших возрастах - в среднем 2-3\%. Средний доход на члена домохозяйства растет с возрастом, но остается тем не менее очень низким и в возрасте 85+, даже за счет повышения пенсий и помощи детей достигает в среднем только 8200 рублей для наименее обеспеченной группы. В основной своей массе эти граждане не получают федеральных и региональных льгот.

В старших возрастных группах от 76 лет значительная доля наименее обеспеченных респондентов имеет уровень образования не выше основного общего; в категории $85+$ половина не имеет даже основного общего образования. Часть вопросов социологического исследования КОУЖ позволяет понять не только источники поступления денежных средств в домохозяйства, их разбивку по источникам дохода (зарплаты, пенсии, помощь детей или социальная помощь), но и структуру потребления респондентов. Показательным индикатором является мобильность (отсутствие мобильности), не обусловленная состоянием здоровья. Среди наименее обеспеченных пожилых граждан, не путешествующих в связи с плохим здоровьем в возрасте 55-65 лет, в восемь раз меньше, чем тех, кто не совершает поездки по причине отсутствия денег. С возрастом этот разрыв сокращается - у пенсионеров ухудшается здоровье как основной фактор благополучия и мобильности, а на отсутствие материальных возможностей жалуются все меньше респондентов.

Наиболее обеспеченная часть пожилого населения (группа 10 \%-10) проживает в городах, имеет более высокий уровень образования, в меньшей степени зависят от фи- 
нансовой и нефинансовой помощи детей как минимум в возрасте до 75 лет. После 76 лет более обеспеченная группа пожилых людей в сравнении с менее обеспеченными имеет меньший процент инвалидности, при том, что количество респондентов, находящихся на диспансерном наблюдении, растет. Вплоть до 76 лет среди наиболее обеспеченных пожилых больше работающих граждан, чем среди наименее обеспеченных, затем с возрастом уровни занятости выравниваются на минимальном уровне.

\section{Дискуссия}

Учитывая тот факт, что экономическая наука в целом вернулась к обсуждению проблем неравенства как не решаемых автоматически в результате экономического роста, системных исследований неравенства именно среди пожилых людей в научной литературе пока не так много. Опыт развитых экономик показывает, что возраст может выступать «всеобщим уравнителем» в случае, когда жизненная траектория человека позволяет ему с возрастом занимать более высокие позиции на рынке труда и получать более высокие доходы, инвестировать свои накопления, формировать финансовую подушку безопасности в течение всей жизни. В такой ситуации уход на пенсию не приводит к резкому падению благосостояния. Напротив, для старшего поколения бывших переходных экономик не только пенсионный период, но и предпенсионный превращаются в борьбу за сохранение рабочего места и уровня дохода как можно дольше, даже в ущерб состоянию здоровья.

\section{Заключение}

Уровень и качество жизни населения в пожилом возрасте определяется не только абсолютными цифрами дохода или относительным материальным благополучием в сравнении с молодым поколением. Наличие и степень неравенства среди пожилых людей в России необходимо оценивать с учетом практически одинаковых стартовых условий в начале жизненного пути современного старшего поколения. Рожденные во времена СССР, представители сегодняшнего поколения 55+ не имели серьезных разрывов в образовании, уровне заработных плат и способе начисления пенсионных выплат внутри возрастных когорт. Тем не менее неравенство среди пожилых людей в России по децильным и квинтильным коэффициентам в 2016-2018 гг. оценивается как трехчетырехкратный разрыв в уровне доходов между наиболее и наименее обеспеченными гражданами. Как свидетельствуют данные, наличие этого разрыва определяется в значительной степени факторами здоровья и, как следствие, способностью работать в возрасте старше пенсионного. Наличие работы, в свою очередь, обеспечивает относительный уровень материальной обеспеченности и независимости от посторонней помощи.

Исследование выполнено за счет гранта Российского научного фонда (проект № 19-18-00282).

\section{СПИСОК ЛИТЕРАТУРЫ}

1. Early J.F. Reassessing the facts about inequality, poverty, and redistribution // Cato Institute Policy Analysis. - 2018. - № 839. URL: https://www.cato.org/sites/cato.org/files/pubs/pdf/pa-839-updated-2.pdf (дата обращения: 21.09.2019).

2. Ngamaba K.H., Panagioti M., Armitage C.J. Income inequality and subjective well-being: a systematic review and meta-analysis // Qual Life Res. - 2018. - № 27 (3). - P. 577-596.

3. Sen A. Poor, relatively speaking // Oxford Economic Papers. - 1983. - № 35. - P. 153-169. URL: https://are.berkeley.edu/courses/ARE251/fall2008/Papers/sen83.pdf (дата обращения: 21.09.2019). 
4. Sen A.K. From income inequality to economic inequality // Southern Economic Journal. - 1997. - V. 64. № 2. - Р. 383-401.

5. Цели ООН в области устойчивого развития. URL: https://www.un.org/sustainabledevelopment/ru/ sustainable-development-goals/ (дата обращения 21.09.2019).

6. Доклад о неравенстве в мире 2018: основные положения. URL: https:/wir2018.wid.world/files/ download/wir2018-summary-russian.pdf (дата обращения 21.09.2019).

7. Schneider S.M. Income inequality and subjective wellbeing: trends, challenges, and research directions // Journal of Happiness Studies. - 2016. - № 17. - P. 1719-1739. DOI: 10.1007/s10902-015-9655-3

8. Капелюшников Р.И. Экономическое неравенство - вселенское зло? - М.: Изд. дом Высшей школы экономики, 2019. - 28 с.

9. Diener Ею, Tay L. Subjective well-being and human welfare around the world as reflected in the Gallup World Poll // International Journal of Psychology. - 2015. - № 50 (2). - P. 135-149. DOI: 10.1002/ijop. 12136

10. Капелюшников Р.И. Феномен старения населения: экономические эффекты. - М.: Институт экономики РАН, 2019. - 50 с.

11. Dupre M.E. Educational differences in age-related patterns of disease: reconsidering the cumulative disadvantage and age-as-leveler hypotheses // Journal of Health and Social Behavior. - 2007. - № 48 (1). - P. 115 .

12. Socioeconomic inequalities in a 16-year longitudinal measurement of successful ageing / A.A.L. Kok, M.J. Aartsen, D.J.H. Deeg, M. Huisman // Journal of Epidemiology and Community Health. - 2016. № 70. - P. 1106-1113.

13. Ahn O., Choi S.H. Predictors of health inequalities in older adults and one-person households during 20102015 // International Journal of Elderly Welfare Promotion and Management. - 2018. - V. 2 (1). - P. $27-32$.

14. Socioeconomic inequalities in resilience and vulnerability among older adults: A population-based birth cohort analysis / T.D. Cosco, R. Cooper, D. Kuh, M. Stafford // International Psychogeriatrics. - 2018. № 30 (5). - P. 695-703.

15. Sheikh M.A., Abelsen B., Olsen J.A. Education and health and well-being: Direct and indirect effects with multiple mediators and interactions with multiple imputed data in Stata // Journal of Epidemiology and Community Health. - 2017. - № 71 (11). - P. 1037-1045.

16. Chen M.-C., Chen K.-M. Economic and living statuses of community-dwelling older adults and the related factors // Geriatrics and Gerontology International. - 2017. - № 17 (10). - P. 1689-1697.

17. Комплексное наблюдение условий жизни населения. 2016. // Федеральная служба государственной статистики. URL: http://www.gks.ru/free_doc/new_site/KOUZ16/index.html (дата обращения 21.09.2019).

18. Комплексное наблюдение условий жизни населения. 2018. // Федеральная служба государственной статистики. URL: https://www.gks.ru/free_doc/new_site/KOUZ18/index.html (дата обращения 21.09.2019).

19. Неравенство и бедность // Федеральная служба государственной статистики. URL: https://www.gks.ru/folder/13723 (дата обращения 21.09.2019).

20. Старшее поколение // Федеральная служба государственной статистики. URL: http://www.gks.ru/wps/ wcm/connect/rosstat_main/rosstat/ru/statistics/population/generation/ (дата обращения 21.09.2019).

Поступила 15.10.2019 2. 
UDC 316.346.32-053.9:314.1

\title{
COMPARATIVE ANALYSIS OF SOCIAL AND DEMOGRAPHIC CHARACTERISTICS OF THE OLDER ADULTS IN RUSSIA
}

\author{
Elena A. Frolova, \\ frolova_ea@mail.tsu.ru \\ Elmira R. Kashapova, \\ elmira6280@yandex.ru \\ Elena I. Klemasheva, \\ klemaha@mail.ru \\ Veronika Malanina, \\ milanskaya@mail.ru
}

\begin{abstract}
National Research Tomsk Polytechnic University, 30, Lenin avenue, Tomsk, 634050, Russia.
\end{abstract}

Elena A. Frolova, Dr. Sc., National Research Tomsk State University, leading researcher, National Research Tomsk Polytechnic University.

Elmira R. Kashapova, laboratory assistant, National Research Tomsk Polytechnic University.

Elena I. Klemasheva, Cand. Sc., laboratory assistant, National Research Tomsk Polytechnic University.

Veronika Malanina, Cand. Sc., associate professor, National Research Tomsk Polytechnic University.

At the turn of the $20^{\text {th }}$ century, economic science and social policy have been revisiting the phenomena of inequality and poverty. However, they are mostly focused on addressing the country's population as a whole. Raising pensions and social benefits, introducing progressive tax system are believed to smooth out the income inequality. Inequality and poverty may not only be instigated by institutional environment, but also by a range of events and individual decisions. The aim of the study is the comparative analysis of social and demographic characteristics of older adults in Russia in the context of income inequality. We also attempt to identify factors correlating with their income status. Methods. We apply the method of decile and quintile income inequality coefficients calculation, as well as Gini index and Palma index calculation for the four age groups of older adults 55+ in Russia. Results. The assessment of inequality indices suggests that inequality coefficients for older adults decrease with age. The groups of the least wealthy and the most wealthy older adults both consist prevalently of women. Above that, especially in young-old ages two income groups have significant differences. The least wealthy live predominantly in rural areas, have lower level of education, employment, mobility and lower self-perceived health compared to the most wealthy.

Key words: Inequality, income inequality, older adults, Gini index, poverty.

The study was supported by the Russian Science Foundation (project №19-18-00282).

\section{REFERENCES}

1. Early J.F. Reassessing the facts about inequality, poverty, and redistribution. Cato Institute Policy Analysis, 2018, no. 839. Available at: https://www.cato.org/sites/cato.org/files/pubs/pdf/pa-839-updated-2.pdf (accessed 21 September 2019). 
2. Ngamaba K.H., Panagioti M., Armitage C.J. Income inequality and subjective well-being: a systematic review and meta-analysis. Qual Life Res., 2018, no. 27 (3), pp. 577-596.

3. Sen A. Poor, relatively speaking. Oxford Economic Papers, 1983, no. 35, pp. 153-169. Available at: https://are.berkeley.edu/courses/ARE251/fall2008/Papers/sen83.pdf (accessed 21 September 2019).

4. Sen A.K. From income inequality to economic inequality. Southern Economic Journal, 1997, vol. 64, no. 2, pp. 383-401.

5. Tseli OON v oblasti ustoychivogo razvitiya [United Nations sustainable development goals]. Available at: https://www.un.org/sustainabledevelopment/ru/sustainable-development-goals/ (accessed 21 September 2019).

6. Doklad o neravenstve v mire 2018: osnovnye polozheniya [World Inequality Report 2018: key points]. Available at: https://wir2018.wid.world/files/download/wir2018-summary-russian.pdf (accessed 21 September 2019).

7. Schneider S.M. Income inequality and subjective wellbeing: trends, challenges, and research directions. Journal of Happiness Studies, 2016, no. 17, pp. 1719-1739. DOI: 10.1007/s10902-015-9655-3

8. Kapelyushnikov R.I. Ekonomicheskoe neravenstvo - vselenskoe zlo? [Economic inequality - universal evil?]. Moscow, Higher School of Economics Publ. house, 2019. 28 p.

9. Diener E., Tay L. Subjective well-being and human welfare around the world as reflected in the Gallup World Poll. International Journal of Psychology, 2015, no. 50 (2), pp. 135-149. DOI: 10.1002/ijop.12136

10. Kapelyushnikov R.I. Fenomen stareniya naseleniya: ekonomicheskie effekty [The phenomenon of population aging: economic effects]. Moscow, Institut ekonomiki RAN Publ., 2019. 50 p.

11. Dupre M.E. Educational differences in age-related patterns of disease: reconsidering the cumulative disadvantage and age-as-leveler hypotheses. Journal of Health and Social Behavior, 2007, no. 48 (1), pp. 1-15.

12. Kok A.A.L., Aartsen M.J., Deeg D.J.H., Huisman M. Socioeconomic inequalities in a 16-year longitudinal measurement of successful ageing. Journal of Epidemiology and Community Health, 2016, no. 70, pp. 1106-1113.

13. Ahn O., Choi S.H. Predictors of health inequalities in older adults and one-person households during 2010-2015. International Journal of Elderly Welfare Promotion and Management, 2018, vol. 2 (1), pp. 27-32.

14. Cosco T.D., Cooper R., Kuh D., Stafford M. Socioeconomic inequalities in resilience and vulnerability among older adults: a population-based birth cohort analysis. International Psychogeriatrics, 2018, no. 30 (5), pp. 695-703.

15. Sheikh M.A., Abelsen B., Olsen J.A. Education and health and well-being: Direct and indirect effects with multiple mediators and interactions with multiple imputed data in Stata. Journal of Epidemiology and Community Health, 2017, no. 71 (11), pp. 1037-1045.

16. Chen M.-C., Chen K.-M. Economic and living statuses of community-dwelling older adults and the related factors. Geriatrics and Gerontology International, 2017, no. 17 (10), pp. 1689-1697.

17. Kompleksnoe nablyudenie usloviy zhizni naseleniya. 2016 [Comprehensive monitoring of living conditions. 2016]. Federalnaya sluzhba gosudarstvennoy statistiki. Available at: http://www.gks.ru/ free_doc/new_site/KOUZ16/index.html (accessed 21 September 2019).

18. Kompleksnoe nablyudenie usloviy zhizni naseleniya. 2018 [Comprehensive monitoring of living conditions 2018]. Federalnaya sluzhba gosudarstvennoy statistiki. Available at: https://www.gks.ru/free_doc/ new_site/KOUZ18/index.html (accessed 21 September 2019).

19. Neravenstvo i bednost [Inequality and poverty]. Federalnaya sluzhba gosudarstvennoy statistiki. Available at: https://www.gks.ru/folder/13723 (accessed 21 September 2019).

20. Starshee pokolenie [Older generation]. Federalnaya sluzhba gosudarstvennoy statistiki [Federal State Statistics Service]. Available at: http://www.gks.ru/wps/wcm/connect/rosstat_main/rosstat/ru/statistics/ population/generation/ (accessed 21 September 2019).

Received: 15 October 2019. 